\title{
ON ZEROS OF SOME ANALYTIC FUNCTIONS RELATED TO THE RIEMANN ZETA-FUNCTION
}

\author{
ANTANAS LAURINČIKAS
}

Vilnius University, Lithuania

\begin{abstract}
For some classes of functions $F$, we obtain that the function $F(\zeta(s))$, where $\zeta(s)$ denotes the Riemann zeta-function, has infinitely many zeros in the strip $\frac{1}{2}<\operatorname{Re} s<1$. For example, this is true for the functions $\sin \zeta(s)$ and $\cos \zeta(s)$.
\end{abstract}

\section{INTRODUCTION}

The zero-distribution of the Riemann zeta-function $\zeta(s), s=\sigma+i t$, is of particular interest in analytic number theory. It is well known that $s=-2 m$, $m \in \mathbb{N}$, are so called trivial zeros of $\zeta(s)$. Moreover, $\zeta(s) \neq 0$ for $\sigma \geq 1$, and for $\sigma \leq 0, t \neq 0$, however, $\zeta(s)$ has infinitely many complex (non-trivial) zeros in the critical strip $\{s \in \mathbb{C}: 0<\sigma<1\}$. The famous Riemann hypothesis $(\mathrm{RH})$ says that all non-trivial zeros of $\zeta(s)$ lie on the critical line $\sigma=\frac{1}{2}$, and this is equivalent to the assertion that $\zeta(s) \neq 0$ for $\sigma>\frac{1}{2}$. At the moment, it is known ([1]) that at least 41 percent of all non-trivial zeros of $\zeta(s)$ in the sense of density lie on the critical line. By numerical calculations [2], the $10^{13}$ first non-trivial zeros are located on the line $\sigma=\frac{1}{2}$. This supports $\mathrm{RH}$.

The best known result on zero-free regions for $\zeta(s)$ is of the form: there exists an absolute constant $c>0$ such that $\zeta(s) \neq 0$ in the region

$$
\sigma \geq 1-\frac{c}{(\log (|t|+2))^{\frac{2}{3}}(\log \log (|t|+2))^{\frac{1}{3}}} .
$$

For the number $N(T)$ of all zeros $\beta+i \gamma$ of $\zeta(s)$ with $0<\beta<1$ and $0<\gamma \leq T$, the von Mangoldt formula

$$
N(T)=\frac{T}{2 \pi} \log \frac{T}{2 \pi \mathrm{e}}+\mathrm{O}(\log T)
$$

2010 Mathematics Subject Classification. 11M41.

Key words and phrases. Riemann zeta-function, universality, zero-distribution. 
is true. These and other classical results on zero-distribution of $\zeta(s)$ can be found in the monograph [4].

On the other hand, there exists zeta-functions similar to $\zeta(s)$ for which the Riemann hypothesis is not true. The simplest example of such functions is the Hurwitz zeta-function $\zeta(s, \alpha)$ with parameter $\alpha, 0<\alpha \leq 1$, defined, for $\sigma>1$, by

$$
\zeta(s, \alpha)=\sum_{m=0}^{\infty} \frac{1}{(m+\alpha)^{s}},
$$

and by analytic continuation elsewhere. However, the functions $\zeta(s)$ and $\zeta(s, \alpha)$ are similar only by their definition by Dirichlet series, and in fact differ one from another very much. The function $\zeta(s, \alpha)$, except for the values $\alpha=1(\zeta(s, 1)=\zeta(s))$ and $\alpha=\frac{1}{2}\left(\zeta\left(s, \frac{1}{2}\right)=\left(2^{s}-1\right) \zeta(s)\right)$, does not have Euler product over primes, and this has a large influence for its properties. The main difference in the zero-distribution problem is that the function $\zeta(s, \alpha)$, $\alpha \neq 1, \frac{1}{2}$, differently from $\zeta(s)$, has zeros in the half-plane $\{s \in \mathbb{C}: \sigma>1\}$, and if $\alpha$ is transcendental or rational $\alpha \neq 1, \frac{1}{2}$, then $\zeta(s, \alpha)$ has infinitely many zeros lying in the $\operatorname{strip}\left\{s \in \mathbb{C}: \frac{1}{2}<\sigma<1\right\}$. More precisely, Theorem 8.4.7 of [6] and [9, Theorem 8, p. 96], says that, for every $\sigma_{1}, \sigma_{2}, \frac{1}{2}<\sigma_{1}<\sigma_{2}<1$, there exists a constant $c=c\left(\alpha, \sigma_{1}, \sigma_{2}\right)>0$ such that, for sufficiently large $T$, the function $\zeta(s, \alpha)$ has more than $c T$ zeros in the rectangle $\left\{s \in \mathbb{C}: \sigma_{1}<\right.$ $\left.\sigma<\sigma_{2}, 0<t<T\right\}$.

The aim of this note is to present some examples of functions $F(\zeta(s))$ for which $\mathrm{RH}$ is not true. This is motivated by a better understanding of the RH problem.

For a region $G$ on the complex plane $\mathbb{C}$, denote by $H(G)$ the space of analytic functions on $G$ endowed with the topology of uniform convergence on compacta. Let $D=\left\{s \in \mathbb{C}: \frac{1}{2}<\sigma<1\right\}$. Define several classes of functions $F$.

$1^{\circ}$ We say that the function $F: H(D) \rightarrow H(D)$ belongs to the class $\operatorname{Lip}(\beta), \beta>0$, if the following hypotheses are satisfied:

a) For every polynomial $p=p(s)$ and every compact subset $K \subset D$ with connected complement, there exists an element $g \in F^{-1}\{p\} \subset H(D)$ such that $g(s) \neq 0$ on $K$;

b) For every compact subset $K \subset D$ with connected complement, there exist a constant $c>0$ and a compact subset $K_{1} \subset D$ with connected complement such that

$$
\sup _{s \in K}\left|F\left(g_{1}(s)\right)-F\left(g_{2}(s)\right)\right| \leq c \sup _{s \in K_{1}}\left|g_{1}(s)-g_{2}(s)\right|^{\beta}
$$

for all $g_{1}, g_{2} \in H(D)$.

Clearly, the set $\{\operatorname{Lip}(\beta): \beta>0\}$ is non-empty. For example, the function $F: H(D) \rightarrow H(D), F(g)=g^{\prime}, g \in H(D)$, is an element of the class Lip $(1)$. This is a simple exercise of using the Cauchy integral formula. 
$2^{\circ}$ Let $S=\{g \in H(D): g(s) \neq 0$ or $g(s) \equiv 0\}$. Denote by $U$ the class of continuous functions $F: H(D) \rightarrow H(D)$ such that, for every open set $G \subset H(D)$, the set $\left(F^{-1} G\right) \cap S$ is non-empty.

We note that the hypothesis that the set $\left(F^{-1} G\right) \cap S \neq \varnothing$ for every open set $G$ is theoretical and with difficulty checked. It can be replaced by a stronger but simpler one.

$3^{\circ}$ Denote by $U_{p}$ the class of continuous functions $F: H(D) \rightarrow H(D)$ such that, for each polynomial $p=p(s)$, the set $\left(F^{-1}\{p\}\right) \cap S$ is non-empty.

An application of the Mergelyan theorem on the approximation of analytic functions by polynomials ([7], see also [10]) shows that $U_{p} \subset U$.

$4^{\circ}$ The main property of the set $S$ is a non-vanishing of functions $g \in$ $H(D)$. The definition of the class $U_{p}$ involves polynomials, however, in the non-bounded region $D$, it is not easy to derive an information on the nonvanishing for the functions $g \in F^{-1}\{p\}$ with a given polynomial $p=p(s)$. Therefore, for $V>0$, we define a bounded region $D_{V}=\left\{s \in \mathbb{C}: \frac{1}{2}<\sigma<\right.$ $1,|t|<V\}$, and in place of the set $S$, take $S_{V}=\left\{g \in H\left(D_{V}\right): g(s) \neq\right.$ 0 or $g(s) \equiv 0\}$.

Denote by $U_{p, V}$ the class of continuous functions $F: H\left(D_{V}\right) \rightarrow H\left(D_{V}\right)$ such that, for each polynomial $p=p(s)$, the set $\left(F^{-1}\{p\}\right) \cap S_{V}$ is non-empty.

It is easily seen that, for some functions $F$ and each polynomial $p=p(s)$, there exists a polynomial $p_{1}=p_{1}(s) \in F^{-1}\{p\}$ and $p_{1}(s) \neq 0$ for $s \in D_{V}$. For example, this holds for the function $F(g)=c_{1} g^{(1)}+\cdots+c_{r} g^{(r)}, \quad g \in$ $H\left(D_{V}\right), \quad c_{1}, \ldots, c_{r} \in \mathbb{C} \backslash\{0\}$.

$5^{\circ}$ For $a_{1}, \ldots, a_{r} \in \mathbb{C}$ and $F: H(D) \rightarrow H(D)$, let $H_{a_{1}, \ldots, a_{r} ; F(0)}(D)=$ $\left\{g \in H(D):\left(g(s)-a_{j}\right)^{-1} \in H(D), j=1, \ldots, r\right\} \cup\{F(0)\}$.

Denote by $U_{a_{1}, \ldots, a_{r} ; F(0)}$ the class of continuous functions $F: H(D) \rightarrow$ $H(D)$ such that $F(S) \supset H_{a_{1}, \ldots, a_{r} ; F(0)}$.

The function $F(g)=g^{N}+a, N \in \mathbb{N}, a \in \mathbb{C}$, clearly, is an element of the class $U_{a ; a}$. The functions $F(g)=\sin g$ and $F(g)=\sinh g$ belong to the class $U_{-1,1 ; 0}$ while the functions $F(g)=\cos g$ and $F(g)=\cosh g$ are elements of the class $U_{-1,1 ; 1}$. To see this, it suffices to solve the equation $F(g)=f$, $f \in H(D)$, in $g \in S$.

$6^{\circ}$ Denote by $\hat{U}$ the class of continuous functions $F: H(D) \rightarrow H(D)$ such that $s-a \in F(S)$ for every $a \in\left(\frac{1}{2}, 1\right)$.

For example, the function $F(g)=g g^{\prime}, g \in H(D)$, belongs to the class $\hat{U}$. To see this, we have to solve the equation

$$
g g^{\prime}=s-a .
$$

Obviously, the latter equation implies

$$
\left(g^{2}\right)^{\prime}=2 s-2 a,
$$

and

$$
g^{2}=s^{2}-2 a s+C,
$$




$$
g= \pm \sqrt{s^{2}-2 a s+C}
$$

with arbitrary constant $C$. We can choose $C$ such that $s^{2}-2 a s+C \neq 0$ for $s \in D$. Thus, there exists $g \in H(D)$ satisfying the equation $F(g)=s-a$. Now we state the theorems on zeros of $F(\zeta(s))$.

ThEOREM 1.1. Suppose that $F$ belongs to at least one of the classes $\operatorname{Lip}(\beta), U, U_{p}, U_{p, V}$ and $\hat{U}$. Then, for every $\sigma_{1}, \sigma_{2}, \frac{1}{2}<\sigma_{1}<\sigma_{2}<1$, there exists a constant $c=c\left(\sigma_{1}, \sigma_{2}, F\right)>0$ such that, for sufficiently large $T$, the function $F(\zeta(s))$ has more than $c T$ zeros lying in the rectangle $\left\{s \in \mathbb{C}: \sigma_{1}<\sigma<\sigma_{2}, 0<t<T\right\}$.

THEOREM 1.2. Suppose that $F$ is an element of the class $U_{a_{1}, \ldots, a_{r} ; F(0)}$, where $\operatorname{Re} a_{j} \notin\left(-\frac{1}{2}, \frac{1}{2}\right), j=1, \ldots, r$. Then the same assertion as in Theorem 1.1 is true.

Proof of Theorems 1.1 and 1.2 is based on the universality of $F(\zeta(s))$.

\section{Universality of $F(\zeta(s))$}

In [8], S. M. Voronin discovered a very interesting approximation property of the function $\zeta(s)$ which now is called universality. He proved that any analytic non-vanishing function can be approximated with a given accuracy uniformly on compact subsets of the strip $D$ by shifts $\zeta(s+i \tau), \tau \in \mathbb{R}$. More precisely, let $K \subset D$ be a compact subset with connected complement, and let $f(s)$ be a continuous non-vanishing function on $K$ which is analytic in the interior of $K$. Then, for every $\varepsilon>0$,

$$
\liminf _{T \rightarrow \infty} \frac{1}{T} \operatorname{meas}\left\{\tau \in[0, T]: \sup _{s \in K}|\zeta(s+i \tau)-f(s)|<\varepsilon\right\}>0 .
$$

Here meas $\{A\}$ denotes the Lebesgue measure of a measurable set $A \subset \mathbb{R}$. Since the approximated functions are non-vanishing, the Voronin theorem does not give any information on the number of zeros of $\zeta(s)$ in the strip $D$. In [5], we began to consider universality theorems for $F(\zeta(s))$ in which the shifts $F(\zeta(s+i \tau))$ approximate not necessarily non-vanishing analytic functions. Thus, theorems of such a kind provide an information on zeros of $F(\zeta(s))$. For the proof of Theorems 1.1 and 1.2, we apply the following universality properties of $F(\zeta(s))$.

LEMma 2.1. Suppose that the function F satisfies the hypotheses at least one of the classes $\operatorname{Lip}(\beta), U$ and $U_{p}$. Let $K \subset D$ be a compact subset with connected complement, and let $f(s)$ be a continuous function on $K$ which is analytic in the interior of $K$. Then, for every $\varepsilon>0$,

$$
\liminf _{T \rightarrow \infty} \frac{1}{T} \operatorname{meas}\left\{\tau \in[0, T]: \sup _{s \in K}|F(\zeta(s+i \tau))-f(s)|<\varepsilon\right\}>0 .
$$


Proof. The case of $U$ was considered in [5]. Since $U_{p} \subset U$, it remains to prove the lemma for the class $\operatorname{Lip}(\beta)$. By the Mergelyan theorem, there exists a polynomial $p=p(s)$ such that

$$
\sup _{s \in K}|f(s)-p(s)|<\frac{\varepsilon}{2} \text {. }
$$

Let $g \in F^{-1}\{p\}$ and $g(s) \neq 0$ on $K$. By the Voronin theorem, the set of $\tau \in \mathbb{R}$ such that

$$
\sup _{s \in K_{1}}|\zeta(s+i \tau)-g(s)|<c^{-\frac{1}{\beta}}\left(\frac{\varepsilon}{2}\right)^{\frac{1}{\beta}}
$$

has a positive lower density. This and $2^{\circ}$ of the class $\operatorname{Lip}(\beta)$ show that the set of $\tau \in \mathbb{R}$ such that

$$
\sup _{s \in K}|F(\zeta(s+i t))-p(s)|<\frac{\varepsilon}{2}
$$

also has a positive lower density what together with (2.1) proves the lemma.

Lemma 2.2. Let $K$ and $f(s)$ be the same as in Lemma 2.1. Suppose that $V>0$ is such that $K \subset D_{V}$, and that $F \in U_{p, V}$. Then the same assertion as in Lemma 2.1 is true.

The lemma in a bit different form is given in [5].

Lemma 2.3. Suppose that the function $F \in U_{a_{1}, \ldots, a_{r} ; F(0)}$. If $r=1$, let $K \subset D$ be a compact subset with connected complement, and let $f(s)$ be a continuous and $\neq a_{1}$ function on $K$ which is analytic in the interior of $K$. If $r \geq 2$, let $K \subset D$ be an arbitrary compact subset and $f \in H_{a_{1}, \ldots, a_{r} ; F(0)}(D)$. Then the same assertion as in Lemma 2.1 is true.

Proof. The lemma for $r=1$ was proved in [5], therefore, we consider the case $r \geq 2$, only. We use a probabilistic limit theorem for $F(\zeta(s))$. Denote by $\mathcal{B}(H(D))$ the $\sigma$-field of Borel sets of the space $H(D)$. Then is known ([4]) that $T^{-1}$ meas $\{\tau \in[0, T]: \zeta(s+i \tau) \in A\}, \quad A \in \mathcal{B}(H(D))$, converges weakly to the probability measure $P_{\zeta}$ on $(H(D), \mathcal{B}(H(D)))$ as $T \rightarrow \infty$, and the support of $P_{\zeta}$ is the set $S$. This and the continuity of $F$ implies that

$$
\frac{1}{T} \operatorname{meas}\{\tau \in[0, T]: F(\zeta(s+i \tau)) \in A\}, \quad A \in \mathcal{B}(H(D)),
$$

converges weakly to $P_{\zeta} F^{-1}$ as $T \rightarrow \infty$.

Let $g \in H_{a_{1}, \ldots, a_{r} ; F(0)}(D)$ be arbitrary. Then we can find $g_{1} \in S$ such that $F\left(g_{1}\right)=g$. This, the continuity of $F$ and the above remarks show that, for every open neighbourhood $G$ of $g$, the inequality $P_{\zeta} F^{-1}(G)>0$ holds. This means that $g$ belongs to the support of the measure $P_{\zeta} F^{-1}$. Thus, the support of $P_{\zeta} F^{-1}$ contains the set $H_{a_{1}, \ldots, a_{r} ; F(0)}(D)$, and even its closure. 
Let

$$
\hat{G}=\left\{g \in H(D): \sup _{s \in K}|g(s)-f(s)|<\frac{\varepsilon}{2}\right\} .
$$

Since $f(s) \in H_{a_{1}, \ldots, a_{r} ; F(0)}(D)$ is an element of the support of $P_{\zeta} F^{-1}$, therefore, $P_{\zeta} F^{-1}(\hat{G})>0$. This together with weak convergence of $(2.2)$ shows that

$$
\liminf _{T \rightarrow \infty} \frac{1}{T} \operatorname{meas}\left\{\tau \in[0, T]: \sup _{s \in K}|F(\zeta(s+i \tau))-f(s)|<\varepsilon\right\} \geq P_{\zeta} F^{-1}(\hat{G})>0 .
$$

Lemma 2.4. Suppose that $F: H(D) \rightarrow H(D)$ is a continuous function, $K \subset D$ is a compact subset, and $f \in F(S)$. Then the same assertion as in Lemma 2.1 is true.

Proof is similar to that of the case $r \geq 2$ of Lemma 2.3.

\section{Proof OF TheOrems}

Proof of Theorem 1.1. We apply Lemmas 2.1, 2.2 and 2.4 with $K=$ $\left\{s \in \mathbb{C}:\left|s-\sigma_{0}\right| \leq \rho\right\}$ and $f(s)=s-\sigma_{0}$, where

$$
\sigma_{0}=\frac{\sigma_{1}+\sigma_{2}}{2} \text { and } \rho=\frac{\sigma_{2}-\sigma_{1}}{2} \text {. }
$$

Then the mentioned lemmas show that, for every $\varepsilon>0$, the set of $\tau \in \mathbb{R}$ satisfying the inequality

$$
\sup _{s \in K}|F(\zeta(s+i \tau))-f(s)|<\varepsilon
$$

has a positive lower density. Now we take $\varepsilon$ such that

$$
0<\varepsilon<\inf _{\left|s-\sigma_{0}\right|=\rho}|f(s)|=\rho .
$$

Then the functions $f(s)$ and $F(\zeta(s+i \tau))-f(s)$ on the disc $K$ satisfy the hypotheses of the classical Rouché theorem. Since the function $f(s)$ has one zero $s=\sigma_{0}$ on $K$, by Rouché's theorem, the sum $F(\zeta(s+i \tau))$ of the functions $F(\zeta(s+i \tau))-f(s)$ and $f(s)$ also has one zero on $K$. However, the measure of $\tau \in[0, T]$ satisfying inequality (3.1), for sufficiently large $T$, is greater than $c T$, and the theorem is proved.

REMARK 3.1. The hypothesis of the class $\hat{U}$ can be replaced by a more general one: there exists a function $g \in S \backslash\{0\}$ such that, for every $a \in\left(\frac{1}{2}, 1\right)$, there exists $b$ with $F(g(b))=0$ and $\operatorname{Re} b=a$. The proof runs in the above way with $f(s)=F(g(s))$ and $K=\left\{s \in \mathbb{C}:\left|s-\sigma_{0}-i t_{0}\right| \leq \rho\right\}$, where $t_{0}$ is such that $F\left(g\left(\sigma_{0}+i t_{0}\right)\right)=0$. 
Proof of TheOREM 1.2. We preserve the notation used in the proof of Theorem 1.1. Since $\operatorname{Re} a_{j} \notin\left(-\frac{1}{2}, \frac{1}{2}\right)$, we have that the function $f(s)=$ $s-\sigma_{0} \neq a_{j}$, in the strip $D, j=1, \ldots, r$. Therefore, the function $f(s)$ on the disc $K$ satisfies the hypotheses of Lemma 2.3, and the further proof runs in the same way as that of Theorem 1.1.

\section{ACKNOWLEDGEMENTS.}

The author thanks an anonymous referee for his suggestion concerning Remark 3.1.

\section{REFERENCES}

[1] H. M. Bui, B. Conrey and M. P. Young, More than $41 \%$ of the zeros of the zeta function are on the critical line, Acta Arith. 150 (2011), 35-64.

[2] X. Gourdon, The $10^{13}$ first zeros of the Riemann zeta function and zeros computation at very large height, http://numbers . computation.free.fr, 2004.

[3] A. Ivić, The Riemann zeta-function, Wiley, New York, 1985.

[4] A. Laurinčikas, Limit theorems for the Riemann zeta-function, Kluwer, Dordrecht, 1996.

[5] A. Laurinčikas, Universality of the Riemann zeta-function, J. Number Theory 130 (2010), 2323-2331.

[6] A. Laurinčikas and R. Garunkštis, The Lerch Zeta-Function, Kluwer, Dordrecht, 2002.

[7] S. N. Mergelyan, Uniform approximations to functions of complex variable, Usp. Matem. Nauk 7 (1952), 31-122 (Russian).

[8] S. M. Voronin, Theorem of the "universality" of the Riemann zeta-function, Izv. Akad. Nauk SSSR. Ser. Mat. 39 (1975), 475-486 (Russian).

[9] S. M. Voronin, Selected works: mathematics, (ed. A. A. Karatsuba), Publishing House MGTU Im. N. E. Baumana, Moscow, 2006 (Russian).

[10] J. L. Walsh, Interpolation and approximation by rational functions in the complex domain, Vol. XX, Amer. Math. Soc. Coll. Publ. 1960.

A. Laurinčikas

Department of Mathematics and Informatics

Vilnius University

Naugarduko 24, LT-03225 Vilnius

Lithuania

E-mail: antanas.laurincikas@mif.vu.lt

Received: 26.3.2012.

Revised: 22.8.2012. 